\title{
Application of mean-variance mapping optimization for parameter identification in real-time digital simulation
}

\author{
Abdulrasaq Gbadamosi \\ Department of Electrical Sustainable Energy, \\ Delft University of Technology, \\ Mekelweg 4, 2628CD, \\ Delft, Netherlands. \\ Email: niyigbada@gmail.com
}

\author{
José L. Rueda, Da Wang, Peter Palensky \\ Department of Electrical Sustainable Energy, \\ Delft University of Technology, \\ Mekelweg 4, 2628CD, \\ Delft, Netherlands. \\ Email: \{j.1.ruedatorres, D.Wang-1, P.Palensky\}@tudelft.nl
}

\begin{abstract}
This paper deals with the process of identifying the parameters of the dynamic equivalent (DE) load model of an active distribution system (ADN) simulated in RTDS using mean-variance mapping optimization (MVMO) algorithm. MVMO is an emerging variant of population-based, evolutionary optimization algorithm whose features include evolution of its solutions through a unique search mechanism within a normalized range of the sample space. Due to the prominent largescale integration of DG in low and medium voltage networks, it is important to develop equivalent models that are suitable for representing the resulting active distribution network in dynamic studies of large power systems. This would significantly reduce the computational demands and simulation time. Moreover, only a defined portion of a system is usually studied, which means that the external system can be substituted with DE thereby allowing the detailed modelling of the focus area. The IEEE 34-Bus distribution system was modified and used as the reference network where measurement data were gathered for identification of the parameters of its developed DE. An optimization-enabled simulation involving MATLAB, which host the MVMO algorithm and RTDS, which simulates the models was established. The reactions of the detailed network and the DE were compared upon subjecting them to different disturbances in the retained system. The effectiveness of the MVMO algorithm in identifying DE parameters based on its unique mapping function is reflected through the results of the response comparison.
\end{abstract}

\section{INTRODUCTION}

$\mathbf{O}$ VER the last couple of years, there has been an increase in the level of renewable energy resources in the electricity grid. Recently, countries such has Germany and Portugal have reportedly supplied most of their energy demands using only renewable energy sources. Consequently, several technical challenges are being faced by utilities with respect to planning and operations of the modern power system. The surge in the capabilities of power electronic devices implies that more large-scale integration of RES such as Wind, PV, Biomass etc. should be expected, especially in $\mathrm{MV} / \mathrm{LV}$ networks. As a result, there is a paradigm shift in the LV networks from traditionally passive to active networks.

Prior to these technological advancement, power systems planners and operators have utilized results from power system stability simulation studies to make appropriate decisions on both short and long-term basis. They use simulations to evaluate the performance and limits of power system components in the network upon subjection to several operating conditions which could compromise the stability of the system. Among all power system components, the need to model the electrical characteristics of loads accurately due to their significant influence on the dynamic behaviour of the power system as long been acknowledged and documented [1], [2], [3]. Besides, a working group (WG) C4.605: "Modelling and aggregation of loads in flexible power networks" was established by CIGRE Study Committee in 2009 to address cogent issues related to load modelling. Since then, they have provided critical and updated overview about the current methodologies and approaches used in load modelling [4].

Most notable among the results of an international survey of utilities done by the work group is the lack of aggregated load models for active distribution networks [5]. Admittedly, it was realized that very few recommendations for dynamic equivalencing of ADN and microgrids exist from the industry. Their preliminary reports suggest that supplementary development of equivalent models for ADNs and MGs be investigated. Moreover, the intermittent nature of the DGs in the active distribution network stresses the need for power system planners to develop adequate models that efficiently represent the grid. These models would facilitate reasonable technical and economic decisions to maintain the stability and reliability of their network.

However, it is a herculean task to build detailed models for such large and multifaceted network due to the computational resources required and the long simulation period. For these reasons, only the specific region of interest (internal network) is usually modelled in detail while the rest of the system (external network) is reduced to equivalent models that provide similar responses [6]. Dynamic equivalent (DE) models are simple aggregated representation of large networks, able to provide similar dynamic responses and behaviours as the actual network for stability analysis. Although developing 
them can be complex, they significantly reduce the simulation time and computational resources.

There are two main steps required to develop adequate DE models. First is to establish the proper structure of the DE based on the characteristic of the region of the network to be reduced. Many methods have been implemented and well documented in literature such as [7]. However, according to [5], only a few have been deployed for ADNs in real time digital simulations. The DE structure that is used in this research is based on recommendations from [8] as shown in Fig. 1. Secondly, a means of identifying the parameters of the defined model is executed such that its responses correlate with those of the reference detailed system upon subjection to the same disturbances. There have been many studies done on developing such aggregate models in several software like PSSE and DigSilent Powerfactory [9] however, only few have been done on models developed in Real Time Digital Simulators. This research contributes to this important field of load modelling by implementing an optimization-enabled real time digital simulation of ADN.

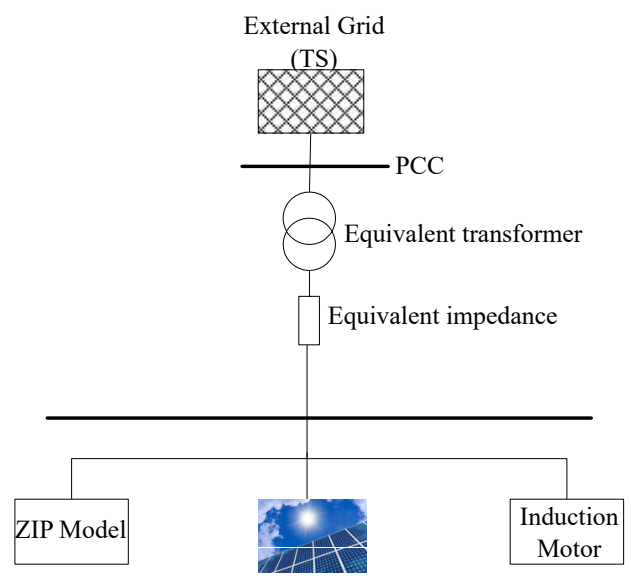

Fig. 1. Dynamic Equivalent load model structure

After establishing the DE structure, reference signal data from the simulated detailed model are used for the identification of appropriate DE parameters. To do this, several optimization techniques based on metaheuristics such as Genetic Algorithms, (GA) [10], Particle swarm optimization (PSO) [11] and Levenberg-Marquardt algorithm (LMA) [12] have been proposed. However, these techniques have some common limitations such as slow convergence, high computational cost, being trapped in a local optimum or low efficiency, with some having better characteristics than others. These problems are due to the non-linear, non-convex and multi-modal nature of the optimization challenge in attempts to properly identify parameters. Nevertheless, due to impressive results of these heuristic-based techniques, this work uses the mean-variance mapping optimization algorithm (MVMO), with its special mapping function described in [13], to determine the parameters of the DE model on RTDS.

The rest of this paper is structured as follows: Section II presents the project approach while the model used are discussed in Section III. The MVMO procedure is elaborated in section IV while the test cases and the associated results are presented in Section VI. Finally, derived conclusions and recommendations for further studies are provided in Section VII.

\section{Proposed ApProACH}

Fig. 2 illustrates the general approach adopted in this research. The reference signals i.e. active and reactive power, were measured at the point of common coupling between the detailed distribution system and the external grid by applying specific disturbances in the external grid. These signals were then stored for subsequent comparison with those measured from the PCC of the DE model. The error between the signals is fed to MVMO algorithm as an objective function. Thereafter, the algorithm supplies new parameters, as a vector $\mathrm{x}$, to the dynamic equivalent model based on its internal evolutionary mechanism. The optimization process stops when the termination criteria is fulfilled. Then the best obtained parameters are updated to the model thus producing a sufficient dynamic equivalent.

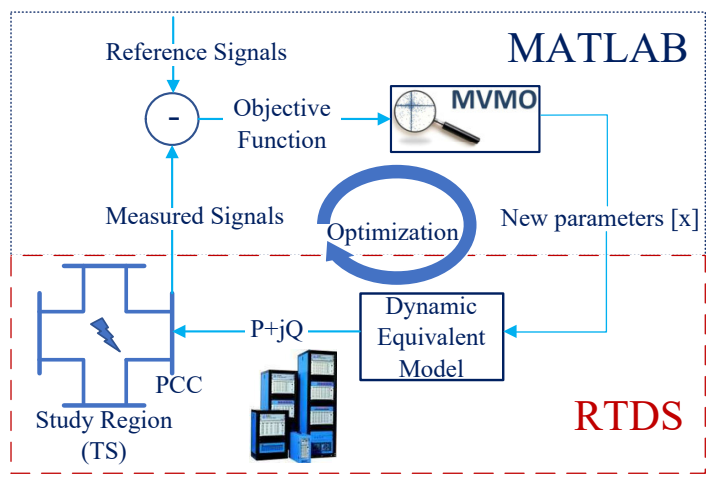

Fig. 2. Research approach

The MVMO optimization algorithm is available as a MATLAB script while the models are built in the RTDS software, RSCAD and simulated in RTDS/Runtime.

\section{DEVELOPED MODELS}

The reference detailed model adopted in this research due to the lack of actual field measurement data is the IEEE 34-Bus distribution system, an actual system in Arizona, provided by the IEEE Power Engineering Society [14]. It operates at a voltage level of $24.9 \mathrm{KV}$ and includes transformers, voltage regulators, shunt capacitors, overhead distribution lines as well as distributed and spot loads which sum up to 1.769MW/1.04MVAR, thus making it an ideal system for this research. However, the system was modified to become an active DS by including PV generation on 3 buses as described in Fig. 3. The modified system was connected to an external transmission system equivalent grid through a transformer and a $120 \mathrm{~km}$ transmission line. In addition, induction motors were also added at a few nodes to increase the contribution of dynamic loads in the detailed reference model. 


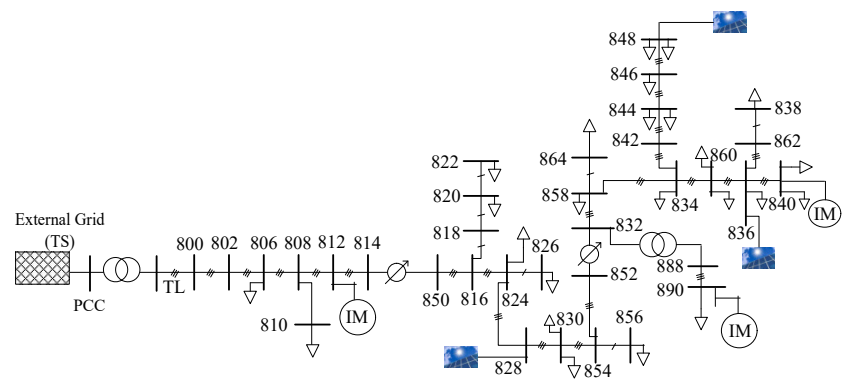

Fig. 3. Modified IEEE 34-Bus reference model

The PV system used for the above modification and also as an aggregate in the DE was modelled using a PV array component in RSCAD component library. The voltage source converter was simulated with small time step in RTDS due to its high switching frequency. The control blocks and the interfacing of the PV array to the grid was implemented as done in [15]. A unique scaling feature is available on the VSC interface transformer which allows the generated power of the PV to be increased without changing any variables. The structure of the DE model was already shown in Fig. 1. The parameters that were chosen for optimization in each block are: the length of the equivalent line, the scale factor and reference voltage (Vsdref) of the PV model, the ZIP percentages of the load on each phase which totals 18 parameters (6 parameters per phase), and 6 parameters of the IM model. In total, 26 parameters were chosen for optimization. These would determine the accuracy of the DE during the period of disturbance.

\section{MVMO-BASEd Solution Procedure}

Fig. 4 illustrates the overall procedure of the MVMO algorithm as implemented in this research. Firstly, the optimization parameters of the model are initialized with their upper and lower bounds. Then the numerical configuration of the algorithm is done. In this case, its settings are as follows: total number of evaluation is 200 , the solution archive size is 4 , number of parameters to be randomly varied is 13 and the scaling factor is set to 1 . Thereafter, the automated phases of the procedure commence with MVMO generating an initial solution vector by randomly sampling the optimization parameters within the defined [min, max] bounds.

Since MVMO's evolution mechanism operates in the normalized search space, the generated values are scaled to the $[0,1]$ range. This search range restricts the algorithm to the defined boundaries. However, the variables are de-normalized before sending them to the RTDS for dynamic simulations and subsequent objective function evaluation. The OF takes as input, the signals stored from the detailed model and those from the simulation of the DE model. Its output is evaluated for fitness and determines the evolution procedure in the inner loop of the flowchart, shaded in Fig. 4.

The inner loop constitutes the core of the algorithm. The solution archive is continuously updated based on the previous outcomes. The best outcome available in memory is chosen as the parent solution from which new solutions (i.e. offspring) are generated. The unique mapping function is also applied to strategically produce new values for an optimization variable set. This phase is known as the mutation phase. The entire optimization procedure is concluded upon fulfilling the termination criterion, which is the specified number of evaluations. An elaborate description of the MVMO algorithm is addressed in [13].

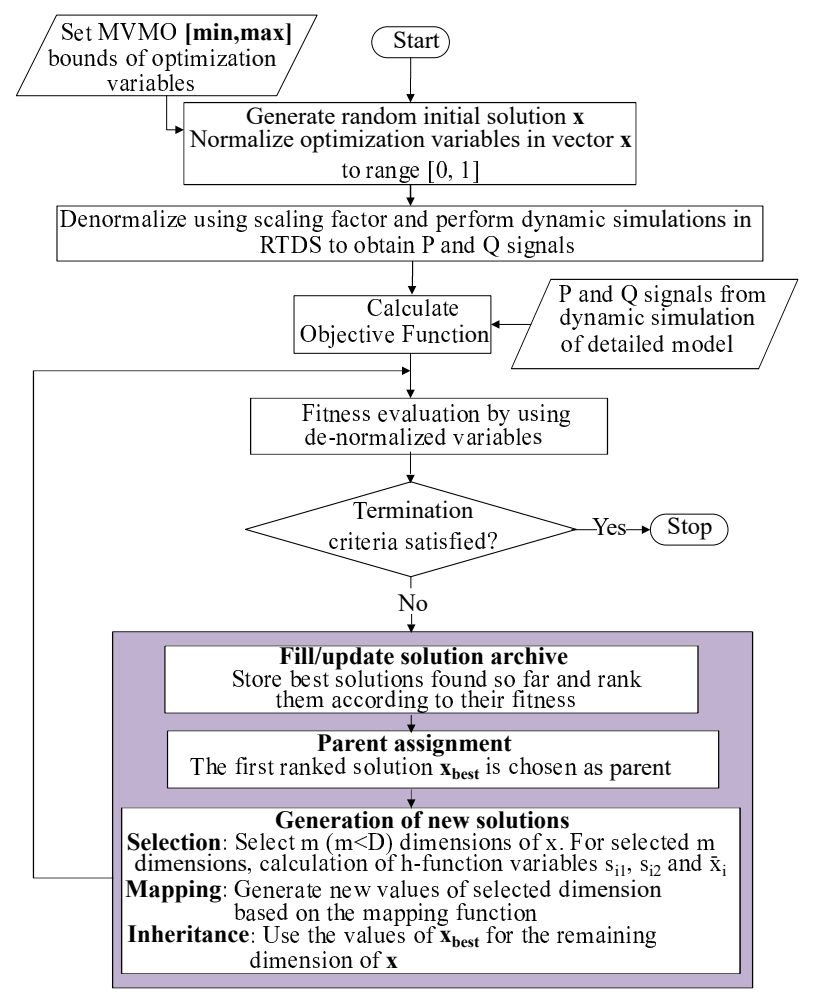

Fig. 4. Flowchart of the approach used for identification of parameters of DE with MVMO

\section{A. Optimization Problem Statement}

The desired goal of the optimization is to derive optimal values of parameters that effect the closest match between the behaviours of the dynamic equivalent model and the detailed model. To do this, the active and reactive power signals are measured at the boundary bus between the external grid and both models. The comparison is formulated as the objective function given in 1 :

Minimize:

$O F=\sum_{n=1}^{p} \sqrt{\alpha_{n} \int_{0}^{\tau}\left[\left(P_{n}-P_{n_{r e f}}\right)^{2}+\left(Q_{n}-Q_{n_{r e f}}\right)^{2}\right] d t}$

Subject to:

$$
x_{\min } \leq x \leq x_{\max }
$$

Where $P_{n}$ and $Q_{n}$ are the active and reactive power signals of the DE, while $P_{n_{\text {ref }}}, Q_{n_{\text {ref }}}$ are the corresponding signals from the detailed model. $\mathrm{p}$ is the number of disturbances, $\alpha_{n}$ 
is the probability of the nth disturbance and $\tau$ is the simulation period. Also, $\mathrm{x}$ is the solution vector that constitutes the set of DE parameters to be optimized while $x_{\min }$ and $x_{\max }$ are the minimum and maximum values defined for each parameter in $\mathrm{x}$. Equation 1 is based on the Euclidean distance function which calculates the point to point distance between two signal vectors. The algorithm aims to reduce this distance error, thus providing very similar response signals.

\section{B. Dynamic Simulation}

Dynamic simulation of the detailed and equivalent models is implemented in RSCAD Runtime environment. To establish a link between RTDS and MATLAB which host the MVMO, a TCP/IP connection is established as described in Fig. 5. A special Runtime script command called 'ListenOnPort()' is used to open a specific communication port (Runtime becomes a server) for MATLAB to connect as a client. Once the connection is established, the port becomes a bi-directional communication channel. Hence, the $\mathrm{P}$ and $\mathrm{Q}$ signals derived at the PCC upon applying a fault in the external grid is sent through this port to MVMO on MATLAB. The OF is calculated using equation 1 and new parameters are sent in the other direction from MATLAB to RTDS/Runtime. Sliders are used in the Runtime module to accept the new de-normalized parameters and a push button component is used to initiate the fault occurrence.

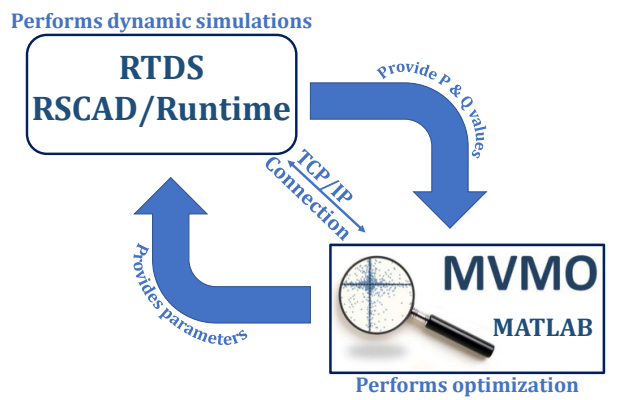

Fig. 5. MATLAB and RSCAD/Runtime Interaction

MATLAB has a jtcp.m program which enables it to send and/or receive TCP packets. Some of the basic functions used in MATLAB to communicate with RTDS/Runtime are detailed below:

- JTCPOBJ = jtcp ( 'REQUEST', Host, Port) represents a request from MATLAB to RTDS/Runtime to establish a TCP/IP connection on the specified port opened by RTDS/Runtime. The host can be represented by an IP address string (e.g. '192.168.0.10') or by a hostname. Since both applications are on the same host, a loopback address ('127.0.0.1') was used. Port is an integer number between 1025 and 65535 which must be open by the server to enable connection.

- jtcp ( ' writes ', JTCPOBJ, msg) sends the specified information contained in the 'msg' variable to RTDS/Runtime through the TCP/IP connection.
- $r m s g=j t c p$ ( ' read', JTCPOBJ) reads the information that is sent from RTDS/Runtime through the communication port and stores it in a variable 'rmsg'.

- jtcp ( 'close', JTCPOBJ) closes the port thereby ending the TCP/IP connection between RTDS/Runtime and MATLAB.

The variable "JTCPOBJ" stores all the necessary information flowing through the communication port which are needed by the remaining functions of the algorithm.

\section{Solution archive}

The solution archive is one of the key features of MVMO algorithm. It serves as the knowledge database which guides the algorithm's search direction. Essentially, the n-best solutions that MVMO has derived at any point in the iteration, with their corresponding fitness value, $d$ factors and shape, are stored in the archive. The archive size is specified at the beginning of the optimization through the main script.

Furthermore, the archive is gradually filled up in a descending order of fitness as the iteration progresses. When the archive is full, it is only updated if a newly generated solution has better fitness than those already stored in the archive. After each update, the mean and shape variables of every optimization parameter $x_{i}$ are calculated using equations 3 and 4 respectively.

$$
\begin{gathered}
\bar{x}_{i}=\frac{1}{n} \sum_{j=1}^{n} x_{i}(j) \\
s_{i}=-\ln \left(v_{i}\right) \cdot f_{s}
\end{gathered}
$$

where the variance $v_{i}$ is computed as follows:

$$
v_{i}=\frac{1}{n} \sum_{j=1}^{n}\left(x_{i}(j)-\bar{x}_{i}\right)^{2}
$$

Initially, $\bar{x}_{i}$ is the same as the randomly generated value of $x_{i}$, and $v_{i}$ is set to 1 . The geometric characteristics of the mapping function is highly influenced by the shape variable $s_{i}$, thus, the reason for $s_{i}$ being dependent on the user defined scaling factor $f_{s}$. Moreover, $s_{i}$ facilitates the control of the mapping function hence the search process.

\section{Evolution of new solutions}

The process of generating new offspring solutions distinguishes MVMO from other algorithms. After the parent vector, $\mathrm{D}$ is chosen, a subset $\mathrm{m}$ out of $\mathrm{D}$ optimization variables are selected for mutation through a random, sequential selection scheme. The mutation is facilitated by the mapping function which samples the random selected dimension $x_{i}$ within the $[0,1]$ limits. The mean and variance of the selected dimension is explored by the function to produce new values. These parameters influence the way the shape of the mapping function varies. As a result, the algorithm's control can switch from a search exploration mode to a search exploitation mode. The mapping function used in this paper based on [13] is as follows: 


$$
\begin{array}{ll}
\frac{\text { if } x_{r}^{*}<0.5}{s_{1}^{*}=s_{1} /(1-\bar{x})} & \frac{\text { if } x_{r}^{*} \geq 0.5}{s_{2}^{*}=s_{2} / \bar{x}} \\
h_{m}=\bar{x}-\frac{\bar{x}}{\left(0.5 \cdot s_{1}^{*}+1\right)} & h_{m}=\frac{(1-\bar{x})}{\left(0.5 \cdot s_{2}^{*}+1\right)} \\
h_{f}=\bar{x} \cdot\left(1-e^{-x_{r}^{*} \cdot s_{1}^{*}}\right) & h_{b}=(1-\bar{x}) /((1- \\
\left.\left.h_{r}^{*}\right) \cdot s_{2}^{*}+1\right)+\bar{x} \\
x_{i}^{\text {new }}=\left(\bar{x}-h_{m}\right) \cdot 2 \cdot x_{r}^{*}+h_{c} & h_{c}=h_{m} \cdot 2\left(1-x_{r}^{*}\right) \\
& x_{i}^{\text {new }}=h_{b}-h_{c}
\end{array}
$$

where $\bar{x}$ is the mean of the selected variable $x_{i}, x_{i}^{n e w}$ represents the new value of the selected dimension $x_{i} . s_{1}^{*}$ and $s_{2}^{*}$ denote the shape factors which vary around measure of entropy as expressed in 5. The entropy measure is a function of the selected variable variance $v_{i}$. The values of $\bar{x}$ and $v_{i}$ are derived from the values available in the solution archive [13].

\section{TEST CASE}

A modified version of the IEEE 34-Bus distribution system was used in this research. PV generators and induction motor were connected to different buses to create an active distribution system and account for industrial dynamic loads respectively. The PV generation accounted for $40 \%$ of the load while the IM added about $15 \%$ additional load. Active (P) and reactive power (Q) signals were measured at the point of common coupling (i.e. HV side of the interfacing transformer) between the external grid and the distribution grid. The measurements were done after three phase faults described in table I were implemented in the external TS grid. Thereafter, these data were stored and used as reference data for validating the parameters of the developed DE load model mentioned in the previous section.

TABLE I

FAULT CASES

\begin{tabular}{|c|c|c|}
\hline $\begin{array}{c}\text { Fault Voltage } \\
(\mathrm{pu})\end{array}$ & $\begin{array}{c}\text { Source Impedance } \\
(\mathrm{ohms})\end{array}$ & $\begin{array}{c}\text { Fault Duration } \\
(\mathrm{ms})\end{array}$ \\
\hline 0.2 & 1.0 & 100 \\
\hline 0.4 & 1.0 & 100 \\
\hline 0.6 & 1.0 & 100 \\
\hline
\end{tabular}

Three fault scenarios were considered in this study. The faults were simulated in the external grid by instantaneously varying the level of the source voltage behind a source impedance. The three-phase source model that was used to represent the external grid in RSCAD has a remote fault feature which allows the faults to be initiated while the simulation is running. However, only three-phase faults can be simulated. The percentage drop in the source voltage during faults represents the occurrence of the faults at various places within the transmission system equivalent grid. The fault duration was set to $100 \mathrm{~ms}$ through the source model configuration menu. The application of fault during every function evaluation was automated through a MATLAB script which sends instruction to RSCAD/Runtime to push the fault button.

\section{NUMERICAL RESUltS}

The simulations were performed on a personal computer with Intel(R) Core(TM) i7-4510U CPU @ 2.0GHz and 8 GB RAM. As mentioned previously, the algorithm was implemented by interfacing MATLAB which performs the optimization with RTDS/Runtime where the dynamic equivalent model is simulated. A special scripting feature in RTDS/Runtime facilitated the communication between both applications. It takes less than a second for MVMO to generate new parameters. However, due to time delays included in the script to allow the model to stabilize in RTDS, it takes approximately 2 minutes to run one iteration of the optimization scheme.

The termination criterion used for the algorithm is the number of evaluations which was set to 200. A fault is applied in the external grid during each evaluation and the error between the power signals of the detailed and DE model is reduced as the iteration progresses. Fig. 6 shows the convergence of MVMO as it attempts to find the least error. It can be observed that MVMO converges quite fast and obtains a nearly optimal solution after about 100 evaluations which is reached within 3.5 hours.

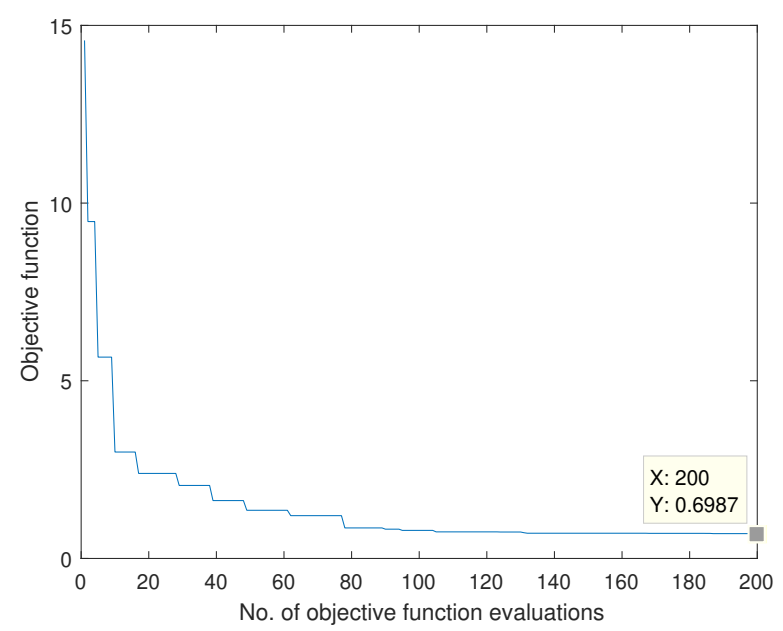

Fig. 6. Convergence plot of MVMO

The value of the objective function is based on the calculation of the Euclidean distance between the active and reactive power signals generated from the detailed and DE models. Since the timestep used for the dynamic simulation in RTDS is about $55 \mu \mathrm{s}$, the data points for 1 second simulation is 18,182 . Therefore, the point to point distance is suitable for determining the difference in the curves. After 200 evaluations, the objective function value was 0.6987 which implies an approximate error reduction of about $95 \%$. 
Fig. 7 and 8 show the result of the first and second fault scenarios. The first fault i.e. 0.2pu was applied during the parameter identification process while the second fault $0.4 \mathrm{pu}$ was applied to the DE model derived from the first scenario as a way of validating the model. From Fig. 7, it can be seen that the DE model produces an identical response to the detailed model response. Besides, similar responses were also derived when a random fault not used during the optimization procedure was applied as shown in Fig. 8.
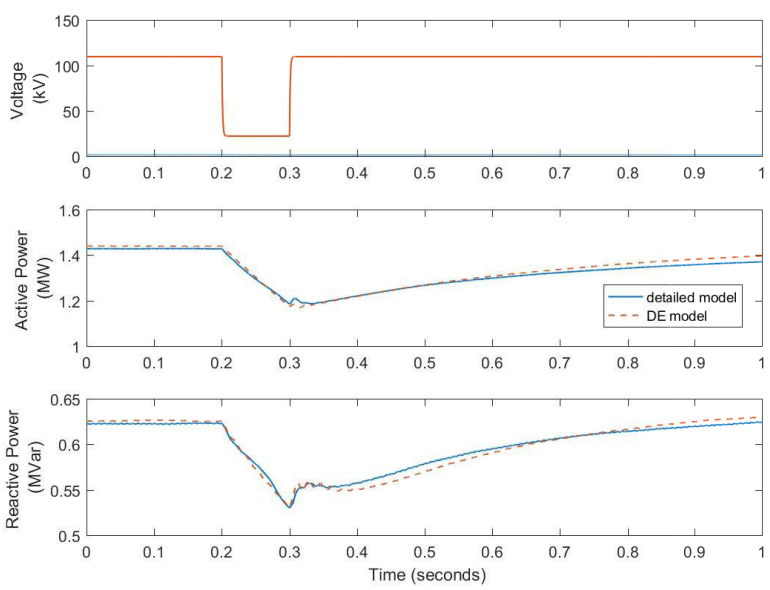

Fig. 7. Fault simulation result for $0.2 \mathrm{pu}$ retained voltage
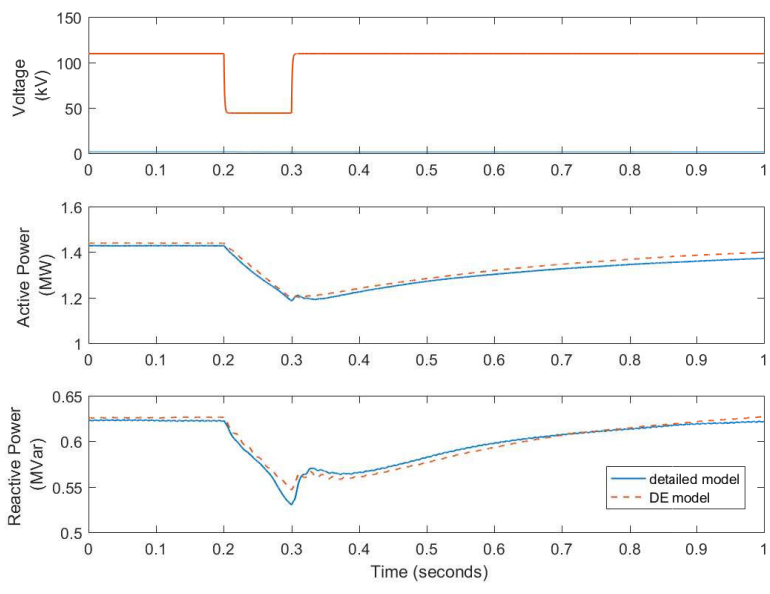

Fig. 8. Fault simulation result for $0.4 \mathrm{pu}$ retained voltage

\section{CONCLUSIONS}

The application of MVMO for identification of dynamic equivalent parameters in Real time digital simulation was presented in this paper. An optimization-enabled real time digital simulation was implemented by connecting MATLAB to RTDS. PV models were included in the detailed model to properly represent an active distribution network. The suitability of the heuristic-based MVMO algorithm was evident through the close similarity of the $\mathrm{DE}$ model reactions to those of the detailed model. Through its unique search and evolutionary mechanism, adequate parameters of the dynamic equivalent model were generated. Therefore, computational resources and simulation time can be reduced by replacing a detailed distribution system with the DE model. The MVMO exhibits fast convergence which proves its effectiveness in reducing the error between the signals measured on the detailed model and the DE model. To further reduce computation time, future research would explore the possibility of implementing parallel computing with the optimization procedure. The inclusion of other DGs to the reference model shall also be considered in subsequent research.

\section{REFERENCES}

[1] IEEE Transactions on Power Systems, "Load representation for dynamic performance analysis of power systems," IEEE Journal 0885-8950, vol. 8, pp. 472-482, May 1993

[2] IEEE Transactions on Power Systems, "Standard load models for power flow and dynamic performance simulation," IEEE Trans 0885-8950, vol. 10, pp. 1302-1313, August 1995

[3] L. Wang, M. Klein, S. Yirga and P. Kundur, "Dynamic reduction of large power systems for stability studies," IEEE Trans 0885-8950,vol. 12, pp. 889-895, May 1997 [IEEE Transactions on Power Systems]

[4] K. Yamashita and S. Djokic and J. Matevosyan and Resende, F. O. and Korunovic, L. M. and Dong, Z. Y. and Milanovic, J. V., "Modelling and aggregation of loads in flexible power networks - Scope and status of the work of CIGRE WG C4.605," IFAC Proceedings Volumes (IFACPapersOnline)|IFAC Proc. Vol. 8, pp. 405-410, 2012.

[5] K. Yamashita, S. M. Villanueva, and J. V. Milanovic, "Initial results of international survey on industrial practice on power system load modelling conducted by CIGRE WG C4.605," in Proc. CIGRE Symp., Bologna, Italy, 2011, vol. C4-333.

[6] J. C. Cepeda, J. L. Rueda and I. Erlich, "Identification of dynamic equivalents based on heuristic optimization for smart grid applications," 2012 IEEE Congress on Evolutionary Computation, Brisbane, QLD, 2012, pp. 1-8. doi: 10.1109/CEC.2012.6256493

[7] Matevosyan J. et al., "Aggregated models of wind-based generation and active distribution network cells for power system studies - literature overview," PowerTech, 2011 IEEE Trondheim, Trondheim, 2011, pp. 18.

[8] Jin Ma, Renmu He and D. J. Hill, "Composite load modeling via measurement approach," IEEE Power Engineering Society General Meeting, Montreal, Que., 2006, pp. 1, doi: 10.1109/PES.2006.1708962

[9] A. M. Azmy and I. Erlich, "Identification of dynamic equivalents for distribution power networks using recurrent ANNs," IEEE PES Power Systems Conference and Exposition, 2004., pp. 348-353 vol.1. doi: 10.1109/PSCE.2004.1397544

[10] C. Kwon and S. D. Sudhoff, "Genetic algorithm-based induction machine characterization procedure with application to maximum torque per amp control," IEEE PES Power Systems Conference and Exposition, pp. 405-415 vol.21., June 2006. doi: 10.1109/TEC.2006.874224

[11] A. Karimi and M. A. Choudhry and A. Feliachi, "PSO-based Evolutionary Optimization for Parameter Identification of an Induction Motor," 39th North American Power Symposium, pp. 659-664, Sept 2007. doi: 10.1109/NAPS.2007.4402380

[12] E. Polykarpou and E. Kyriakides, "Parameter estimation for measurement-based load modeling using the Levenberg-Marquardt algorithm," 18th Mediterranean Electrotechnical Conference (MELECON), pp. 1-6, April 2016. doi: 10.1109/MELCON.2016.7495363

[13] J. L. Rueda, I. Erlich, "MVMO for bound constrained single-objective computationally expensive numerical optimization," IEEE Congress 1089-778X, pp. 1011-1017, May 2015 [IEEE Congress on Evolutionary Computation (CEC)]

[14] IEEE 34 Node Test Feeder, "Distribution System Analysis Subcommittee of the IEEE Power Engineering Society," http://www.ewh.ieee.org/ soc/pes/dsacom/testfeeders/, Accessed: 2016-12-30

[15] O. Nzimako and A. Rajapakse, "Real time simulation of a microgrid with multiple distributed energy resources," International Conference on Cogeneration, Small Power Plants and District Energy (ICUE), Bangkok, 2016, pp. 1-6. doi: 10.1109/COGEN.2016.7728945 